Supplement materials:

\title{
Disruption of BSEP Function in HepaRG cells Alters Bile Acid Disposition and is a susceptive factor to Drug-Induced liver
} Injury

Xi Qiu, Yueping Zhang, Tongtong Liu, Hong Shen, Yongling Xiao ${ }^{1}$, Maureen Bourner ${ }^{1}$, Jennifer Pratt $^{1}$, David Thompson ${ }^{1}$, Punit Marathe, W. Griffith Humphreys and Yurong Lai*

Pharmaceutical Candidate Optimization, Bristol-Myers Squibb, 3551 Lawrenceville Road, Princeton, NJ 08540

${ }^{1}$ Sigma-Aldrich, Life Science and Technology Center, Sigma-Aldrich, St. Louis, MO 63103

Supplement Table 1. Parameters of mass spectrometry for Bile acids

\begin{tabular}{|l|l|l|l|l|l|l|l|}
\hline Bile acids & Abbreviation & MW & Q1 & Q3 & RT & CID & DP \\
\hline Cholic acid & CA & 408 & 407.1 & 343.1 & 1.53 & -44 & -100 \\
\hline Chenodeoxycholic acid & CDCA & 392 & 391.3 & 391.3 & 1.77 & -10 & -90 \\
\hline Taurochenodeoxycholic acid & TCDCA & 499 & 498.3 & 79.9 & 1.81 & -110 & -130 \\
\hline Deoxycholic acid & DCA & 392 & 391.3 & 345.2 & 1.84 & -45 & -160 \\
\hline Glycocholic acid & GCA & 465 & 464.3 & 74 & 1.56 & -60 & -90 \\
\hline glycodeoxycholic acid & GDCA & 449 & 448.3 & 74 & 1.86 & -60 & -90 \\
\hline Glycochenodeoxycholic acid & GCDCA & 449 & 448.3 & 74 & 1.79 & -60 & -90 \\
\hline Taurocholic acid & TCA & 515 & 514.3 & 80 & 1.59 & -130 & -120 \\
\hline Taurodeoxycholic acid & TDCA & 499 & 498.3 & 79.9 & 1.88 & -110 & -130 \\
\hline Taurochenodeoxycholic Acid & TCDCA & 499 & 498.3 & 79.9 & 1.81 & -110 & -130 \\
\hline Ursodeoxycholic acid & UDCA & 392 & 391.3 & 391.3 & 1.38 & -10 & -90 \\
\hline Tauroursodeoxycholic acid & TUDCA & 499 & 498.3 & 80 & 1.43 & -110 & -130 \\
\hline Glycoursodeoxycholic acid & GUDCA & 449 & 448.3 & 74 & 1.39 & -60 & -90 \\
\hline
\end{tabular}




\begin{tabular}{|l|l|l|l|l|l|l|l|} 
Deuterated dexoycholic acid & $\mathrm{D}_{4}$-DCA & 396.5 & 395.2 & 349.1 & 1.84 & -45 & -160 \\
\hline
\end{tabular}


Supplement Table 2 Proteotypic peptides for transporter quantification by a LC-MS/MS

\begin{tabular}{|c|c|c|c|c|c|c|c|c|}
\hline \multirow[b]{2}{*}{ Transporter } & \multicolumn{4}{|c|}{ Quantitation Peptides } & \multicolumn{4}{|c|}{ Isotopic internal Standards } \\
\hline & Sequence & $\begin{array}{l}\text { molecular } \\
\text { weight }\end{array}$ & Q1 & Q3 & $\begin{array}{l}\text { Sequence (*isotope labeled } \\
\text { amino acid) }\end{array}$ & $\begin{array}{l}\text { Molecular } \\
\text { weight }\end{array}$ & Q1 & Q3 \\
\hline \multirow{3}{*}{ OATP1B1 } & \multirow{3}{*}{ NVTGFFQSFK } & 1176.0 & 587.8 & 961.4 & \multirow{3}{*}{ NVTGFFQSFK* } & \multirow[t]{3}{*}{1184.0} & \multirow[t]{3}{*}{591.8} & 969.4 \\
\hline & & & 587.8 & 860.4 & & & & 868.4 \\
\hline & & & 587.8 & 656.3 & & & & 664.3 \\
\hline \multirow{4}{*}{ OATP1B3 } & \multirow{4}{*}{ NVTGFFQSLK } & 1140.0 & 570.8 & 927.4 & \multirow{4}{*}{ NVTGFFQSL*K } & 1149.0 & 574.3 & 934.4 \\
\hline & & & 570.8 & 826.4 & & & & 833.5 \\
\hline & & & 570.8 & 622.3 & & & & 629.3 \\
\hline & & 1595.0 & 799.1 & 712 & & 1606.0 & 802.9 & 715.9 \\
\hline \multirow[t]{3}{*}{ OATP2B1 } & \multirow[t]{3}{*}{ SSPAVEQQLLVSGPGK } & & 798.9 & 445.2 & \multirow[t]{2}{*}{ SSPAVEQQLLVSGPGK* } & & & 453.2 \\
\hline & & & 798.9 & $\begin{array}{l}1155 . \\
5\end{array}$ & & & & 1163.6 \\
\hline & & 880.5 & 441.0 & 423.6 & & 887.0 & 445.1 & 431.6 \\
\hline \multirow[t]{3}{*}{ ост1 } & \multirow[t]{3}{*}{ ENTIYLK } & & 441.0 & 637.7 & \multirow[t]{3}{*}{ ENTIYLK* } & & & 645.8 \\
\hline & & & 441.0 & 536.6 & & & & 544.5 \\
\hline & & 942.5 & 472.0 & 559.4 & & 951.0 & 476.8 & 569.8 \\
\hline \multirow[t]{3}{*}{ ОСТ3 } & \multirow[t]{3}{*}{ GPSAAALAER } & & 472.0 & 488.8 & \multirow[t]{3}{*}{ GPSAAALAER* } & & & 498.7 \\
\hline & & & 472.0 & 630.9 & & & & 640.8 \\
\hline & & 1044.0 & 522.9 & 757.3 & & 1054.0 & 527.9 & 654.3 \\
\hline \multirow[t]{3}{*}{ BCRP } & \multirow[t]{3}{*}{ SSLLDVLAAR } & & 522.9 & 644.3 & \multirow[t]{3}{*}{ SSLLDVLAAR* } & & & 767.3 \\
\hline & & & 522.9 & 870.3 & & & & 880.3 \\
\hline & & 1269.7 & 635.3 & 972.4 & & 1278.0 & 640.3 & 981.4 \\
\hline \multirow[t]{3}{*}{ P-gp } & \multirow[t]{3}{*}{ AGAVAEEVLAAIR } & & 635.3 & 900.3 & \multirow[t]{3}{*}{ AGAVAEEVLAAIR* } & & & 910.3 \\
\hline & & & 635.3 & 771.3 & & & & 781.3 \\
\hline & & 1029.0 & 515.4 & 841.7 & & 1036.0 & 518.8 & 848.5 \\
\hline BSEP & STALQLIQR & & 515.4 & 657.5 & STALQL*IQR & & & 664.4 \\
\hline & & & 515.4 & 770.4 & & & & 777.4 \\
\hline & & 1770.0 & 885.8 & 665.5 & & 1778.0 & 889.8 & 669.5 \\
\hline MRP2 & LTIIPQDPILFSGSLR & & 885.8 & 989.4 & LTIIPQDPILFSGS*LR & & & 997.5 \\
\hline & & & 885.8 & 441.2 & & & & 445.2 \\
\hline & & 1620.9 & 541.6 & 697.2 & & 1630.0 & 544.5 & 702.6 \\
\hline MRP3 & IDGLNVADIGLHDLR & & 541.6 & 755.1 & IDGLNVADIGLHDLR* & & & 759.8 \\
\hline & & & 541.6 & 612.1 & & & & 617.1 \\
\hline & & 965.4 & 482.8 & 796.4 & & 975.4 & 487.8 & 806.4 \\
\hline MRP4 & APVLFFDR & & 482.8 & 697.4 & APVLFFDR* & & & 707.4 \\
\hline & & & 482.8 & 584.3 & & & & 594.3 \\
\hline NTCP & GIYDGDLK & 880.0 & 440.8 & 710.2 & GIYDGDIK* & 888.0 & 444.8 & 718.2 \\
\hline & & & 440.8 & 547.3 & & & & 555.3 \\
\hline
\end{tabular}


Figure 1. Time-dependent accumulation of BAs in HepaRG WT and KO cells exposed to BSEP inhibitors, troglitazone, bosentan and dipyridamole. On day 4, HepaRG KO and WT cells were cultured with medium containing BSEP inhibitors, troglitazone ( 5 or $50 \mu \mathrm{M})$, bosentan ( 5 or $50 \mu \mathrm{M})$ and dipyridamole ( 3 or $30 \mu \mathrm{M})$. At designated time points $(0,2,4,8$ and $24 \mathrm{hr}$ ), the cells were washed with ice cold HBSS buffer and BAs in the cells were extracted. Individual BA were analyzed by LC-MS/MS. Data are shown as mean $\pm S D(n=3)$. Student's $t$ test was used to denote the significant increase of intracellular BA between at $0 \mathrm{hr}$ and $24 \mathrm{hr}$ from the same treatment $\left({ }^{*} \mathrm{P}<0.05\right)$, or between the treated cells and untreated cells ( $\left.{ }^{\#} \mathrm{P}<0.05\right)$.

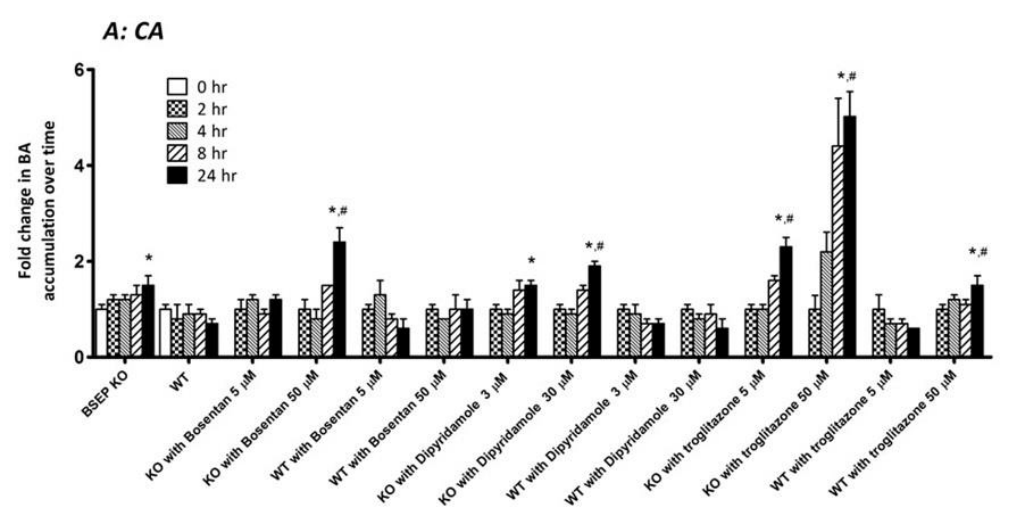



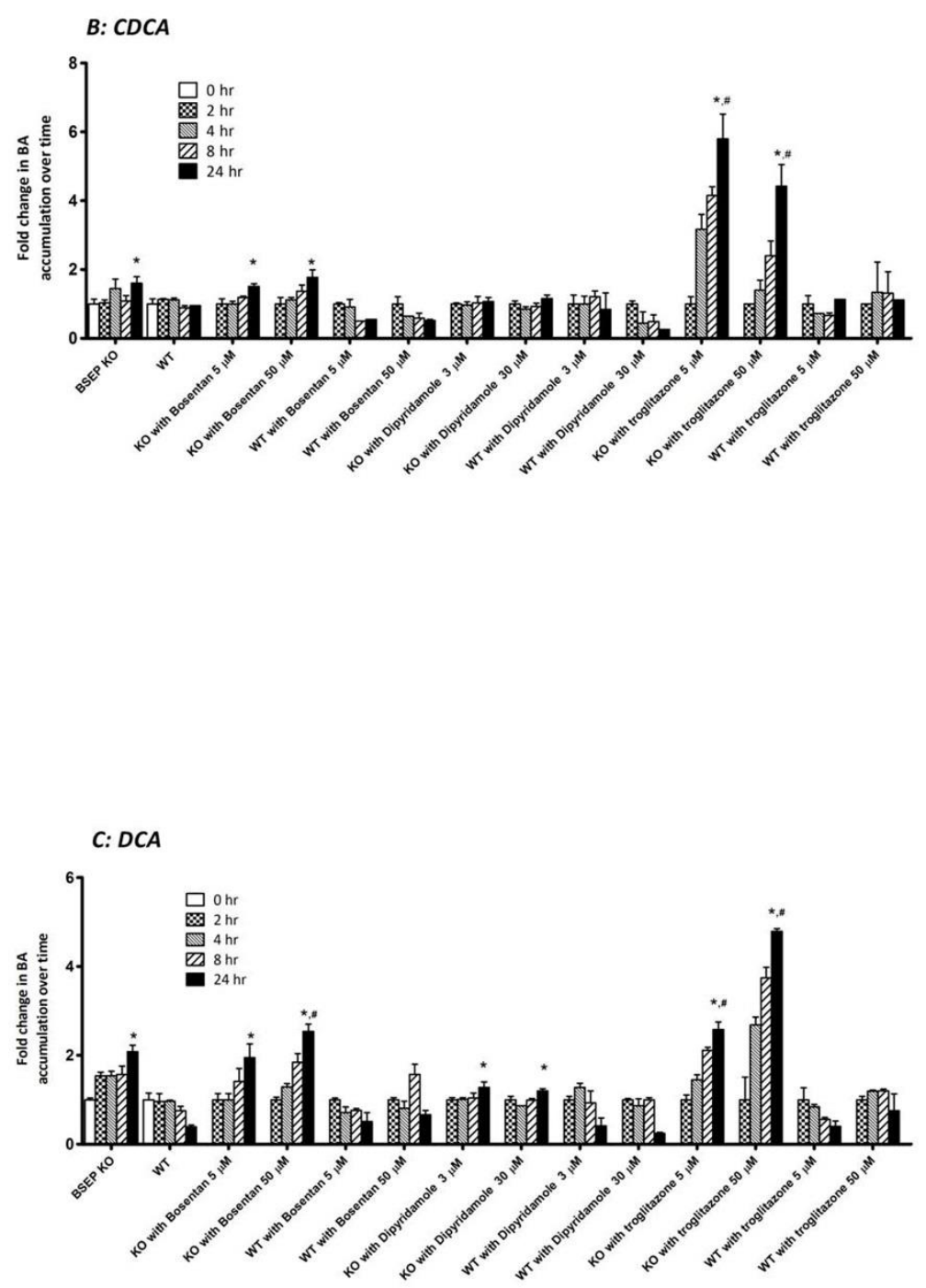

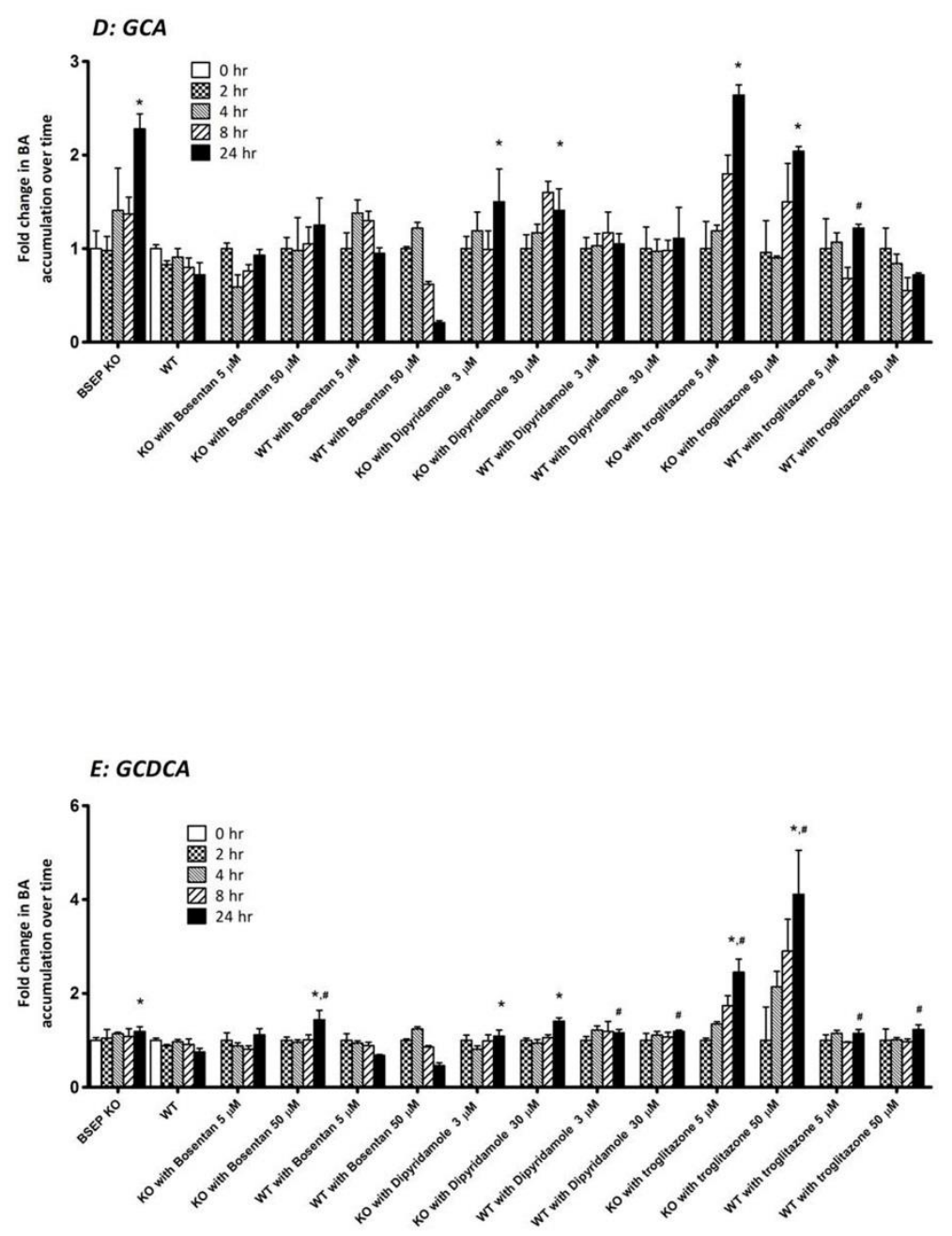

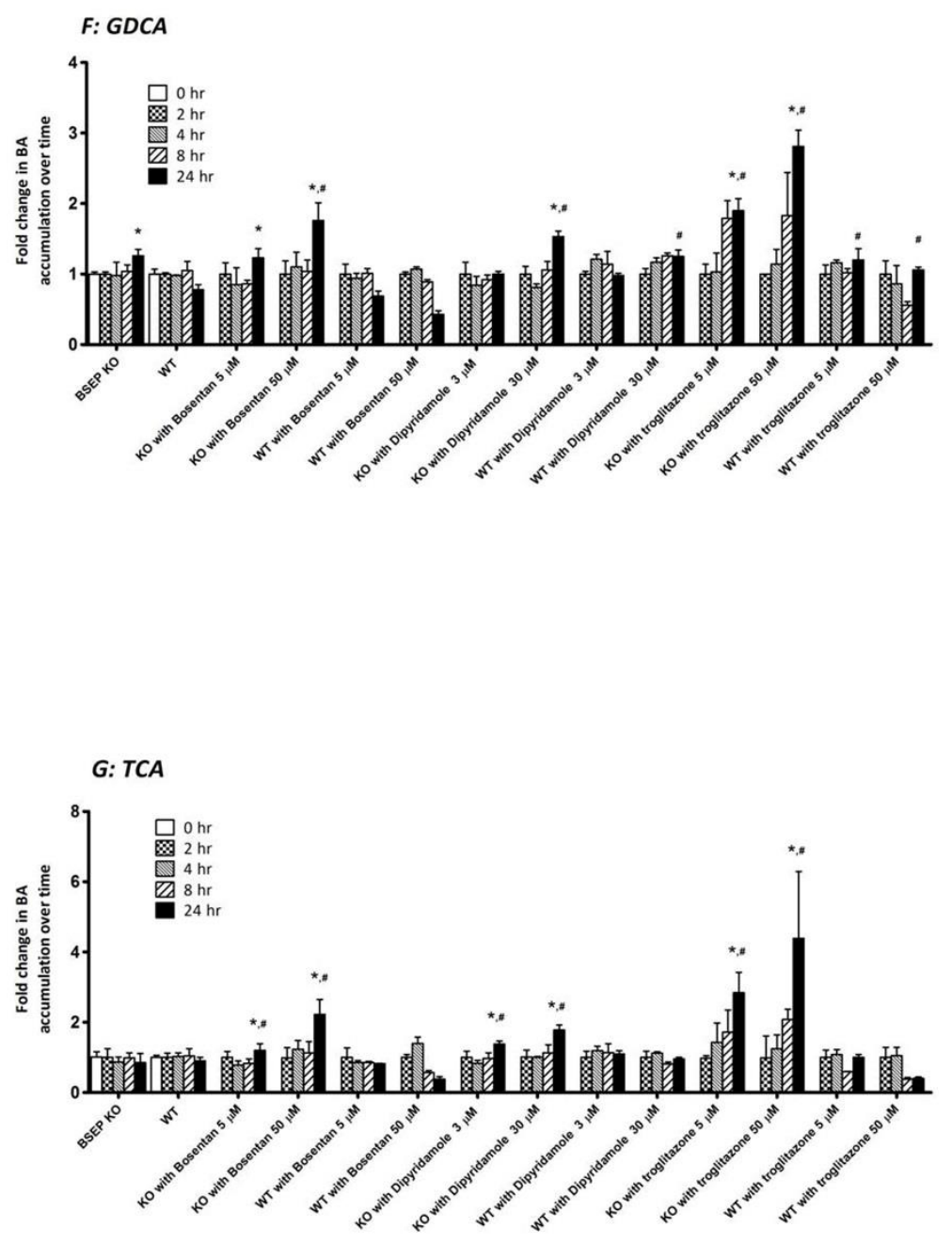

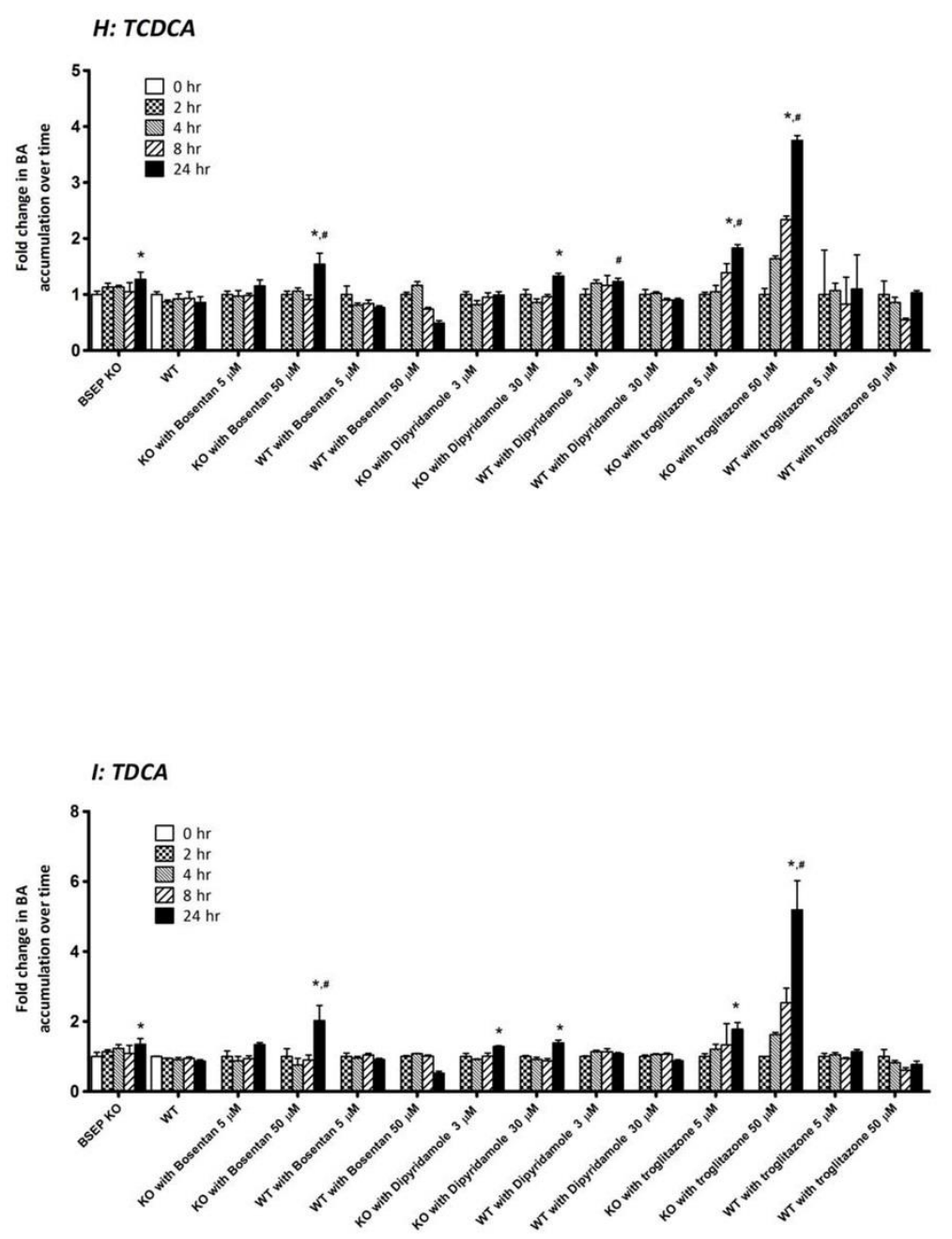


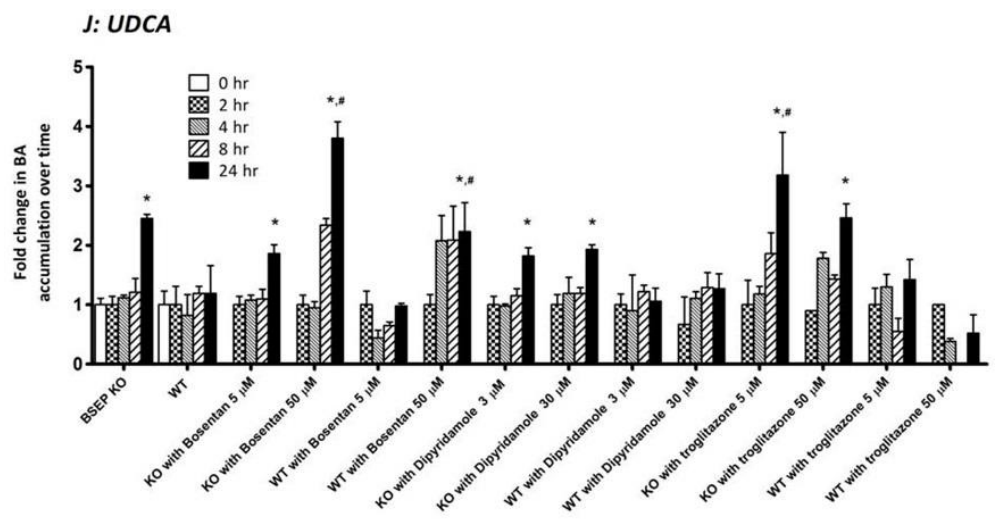

